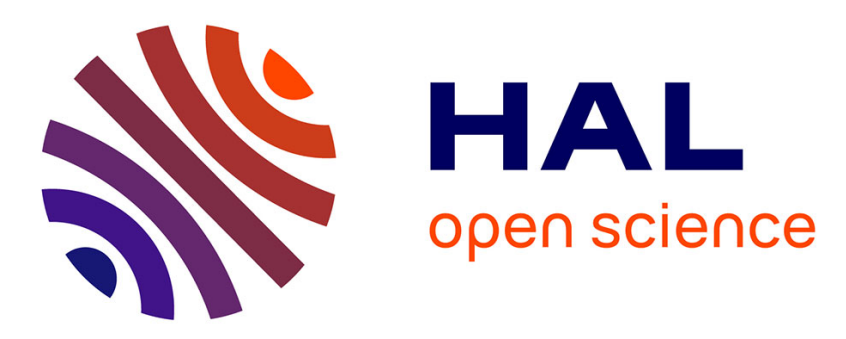

\title{
Human identification system based on the detection of optical Disc Ring in retinal images
}

\author{
Takwa Chihaoui, Rostom Kachouri, Hejer Jlassi, Mohamed Akil, Kamel
}

Hamrouni

\section{- To cite this version:}

Takwa Chihaoui, Rostom Kachouri, Hejer Jlassi, Mohamed Akil, Kamel Hamrouni. Human identification system based on the detection of optical Disc Ring in retinal images. 5th International Conference on Image Processing Theory, Tools and Applications, Nov 2015, Orléans, France. pp.263267, 10.1109/IPTA.2015.7367143 . hal-01309990

\section{HAL Id: hal-01309990 https://hal.science/hal-01309990}

Submitted on 1 May 2016

HAL is a multi-disciplinary open access archive for the deposit and dissemination of scientific research documents, whether they are published or not. The documents may come from teaching and research institutions in France or abroad, or from public or private research centers.
L'archive ouverte pluridisciplinaire HAL, est destinée au dépôt et à la diffusion de documents scientifiques de niveau recherche, publiés ou non, émanant des établissements d'enseignement et de recherche français ou étrangers, des laboratoires publics ou privés. 


\title{
Human Identification System based on the detection of Optical Disc Ring in retinal images
}

\author{
Takwa Chihaoui ${ }^{12}$, Rostom Kachouri ${ }^{2}$, Hejer Jlassi ${ }^{1}$, Mohamed Akil ${ }^{2}$ and Kamel Hamrouni ${ }^{1}$ \\ ${ }^{1}$ Universite de Tunis El Manar, Ecole Nationale d'Ingenieurs de Tunis, LR-SITI Signal Image et Technologie de l'information, Tunis, Tunisie \\ e-mail: hejer_enit@enit.rnu.tn, kamel.hamrouni@enit.rnu.tn \\ ${ }^{2}$ Universite Paris-Est, Laboratoire Informatique Gaspard-Monge, Equipe A3SI, ESIEE Paris, France \\ e-mail:takwa.chihaoui@esiee.fr, rostom.kachouri@esiee.fr, mohamed.akil@esiee.fr
}

\begin{abstract}
Retinal identification is being studied as a hot research topic in the biometric field. Many factors such as the poor quality of retinal images and the huge execution time can seriously affect the performance of the retinal identification systems. In this context, in order to compromise the quality and the processing time, this paper presents a human identification system based on a new preprocessing method of retinal images that we call optical Disc Ring detection method. The proposed ODR method consists of three phases: image quality improvement in order to enhance the contrast of input retinal image, the optical disc center detection and the extraction of an interest ring around the detected center which contains the densest retinal information. This proposed method was integrated in an identification system based on SIFT features and evaluated using a subset of the VARIA retinal database. The correct identification rate was found to be $99.89 \%$ while the reached execution time is very lower than other existing identification systems.
\end{abstract}

Keywords-Biometrics, retinal images, preprocessing, image enhancement, Optical Disc Ring (ODR) extraction, Scale Invariant Feature Transform (SIFT) descriptors.

\section{INTRODUCTION}

Nowadays, with the advance in technology, biometrics becomes an improved interest for people identification or authentification. Indeed conventional personnel authentification systems based on map ID or password are vulnerable. It is very easy to forge an identity card or to imitate a signature. A solution to these problems has been found in the technology based on biometric authentification. Biometrics is an emerging field of technology using distinguishing and measurable morphological and behavioral characteristics that can be processed to established the automatic recognition and identification of individuals. In fact, behavioral biometrics is based on the analysis of certain behavior of a person such as voice, signature and keystroke. However, morphological biometrics includes fingerprint, palmprint, hand geometry, vein, face, iris, retina and ear. The eye offers the most reliable biometric features which are the iris and the retina [1]. The iris has enjoyed great interest from researchers and many biometric systems based on the iris are installed in secure locations such as airports [2]. On the other hand, because of the difficulty of the fundus image acquisition, the retina has not had an important interest in the beginning. As known to be very reliable and difficult to forge, Carelton Simon and Greg Heacok [3] were interested in the biometrics by the retina and had marketed
2002 by the first prototype of the retina device acquisition. This retina recognition is an automatic method based on a robust and distinctive feature in order to identify individual or verify its claimed identity [5]. Indeed, the uniqueness of the retina blood vessels is not easy to replicate or forge; in addition it is free from the surroundings effects. In this context, it can be considered as the most accurate among the unique, universal and time-invariant patterns. However, personal identification systems based on retina can suffer from some problems. On the one hand, due to the acquisition process of the retinography, retinal images often suffer from imperfections such as poor gray level contrast and background intensity variation. These defects can seriously affect features extraction and reduce the retinal identification rate as consequence. On the other hand, the retina has a dense structure of vessel information which increases the execution time of identification systems. In order to overcome these problems, we propose in this paper a new and robust method to preprocess retinal images. It aims principally to improve the quality of input images and reduce the processing time of the identification system. The identification task in the proposed system is based on SIFT description, which is invariant to the affine transformations such as rotation, scale changes and translation. This paper is organized under five sections as follows: Section 2 presents state of the art for retinal image preprocessing. In section 3, the proposed method is explained and its general flowchart is described. Section 4 presents the experimental results and discussion. Finally, conclusion is drawn in last section.

\section{STATE OF THE ART}

The automatic analysis of retinal images is a difficult task because images taken at standard examinations could contain artifacts and are generally poorly contrasted. Non-uniformity of illumination raises the intensity levels in some regions of an image, while other regions farther away from the optical disc may suffer from a reduction of brightness. In this context, various methods have been proposed in order to improve the quality of retinal images. Existing methods that focus on the preprocessing of retinal images can be generally grouped as: Vessel extraction-based methods and improved quality of retinal image-based methods. 


\section{A. Vessel extraction-based methods}

For this class, the aim is to obtain a binary image as an input for feature extraction step. For example, in their personal recognition system, Ortega et al. [11] employed a fuzzy circular Hough transform to localize the optical disc in the retina image. They obtain as a result of preprocessing a crease model of the retinal vessels inside the optical disc. This method may give a false detection of optical disc. In order to improve and reduce the false localization of optical disc, Tabatabaee et al. [10] proposed a biometric system based on a new approach of preprocessing by localizing the optical disc using Haar wavelet and snake model. The most problem of this method is that the optical disc localization strongly depends on the selection of the initial contour active. Indeed, the choice of the initial contour is very important to reduce the false optical disc detection. To avoid the experimental involvement, Farzin et al. [14] proposed an automatic preprocessing method based on a local contrast enhancement process. This method consisted of four steps: First, the optical disc of the retina is located using a template matching technique. Therefore, they employed a correlation technique in order to delete undesired brightness effect of the optical disc. Then, a contrast enhancement method based on statistical properties is ensured. Finally,an histogram thresholding is used to obtain a binary image containing blood vessel. The information extracted from the vascular tree is generally incomplete because of the convergence of multiple plies. Also, some micro vascular segments can not be extracted effectively. Hannu Oinonen et al. [8] used the multiscale enhancement approach proposed by Frangi et al. [12] in order to overcome the problem of lack of some micro vascular information. Then, the p-tile thresholding is applied to extract binary images. The major drawback of this class is that the output binary image may suffer from the discontinuity of some vascular segments which can affect the feature extraction and cause mismatching.

\section{B. Improved quality of retinal image-based methods}

This class focuses on the improvement of retinal image without using a binarization process. Marn et al. [6] proposed a preprocessing method for blood vessel segmentation. It is based on a background homogenizing method. They estimated the background of the retina by a filtering operation with a large arithmetic mean kernel. Xianjing Meng et al. [13] proposed a new system for identifying people by the retina based on an advanced circular Gabor filter tool and the iterated spatial anisotropic smooth method in order to reduce the noise in homogeneous regions. It is an efficient method but it suffers from an important execution time. Hejer Jlassi et al. [9] used the top-hat transform with a linear structuring element at different orientations in order to enhance the vascular tree followed by a contrast enhancement as a preprocessing step. The most problem of this category is that is very timeconsuming. In order to overcome these problems, we are going to propose a new preprocessing method which enhance the retinal image and extract the region with the high density of vessel information. Segmentation and binary vessels extraction are not needed in this approach. So, the computational complexity of the image matching algorithm is low compared to other recent identification systems.

\section{PROPOSED ODR METHOD}

The optical disc is the brightest area in the digital retinal images where blood vessels converge. Its detection is very important since it is the core of vessel information. We propose a method for preprocessing the retinal image that we call ODR method by determining the position of the optical disc, a threestep algorithm is used: fist, the contrast-Limited Adaptative Histogram equalization is applied in order to enhance the entire input image. Then, the sauvola binarisation is employed in order to detect the center of the most brilliant region of the retina. The final step consists of extract a ring interest around the detected center to be served as an input image for SIFT feature generation. The different designed steps are well described in the figure 1 .

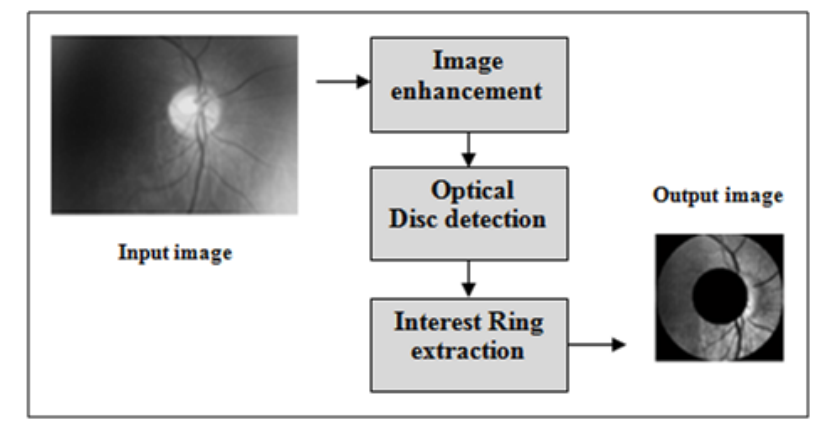

Fig. 1. Flow Chart of our ODR method.

\section{A. Image quality enhancement}

Due to the acquisition process of the retinography, retinal images may present lighting defects and background intensity variation.

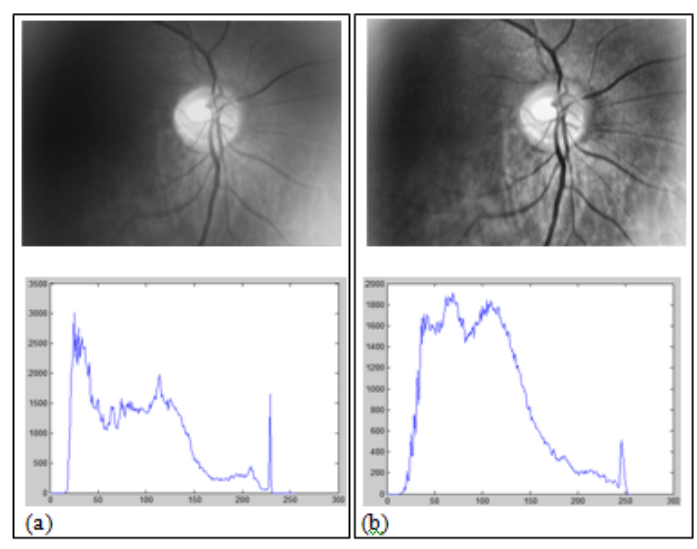

Fig. 2. Contrast enhancement results: histogram (a) input image and (b) enhanced image. 


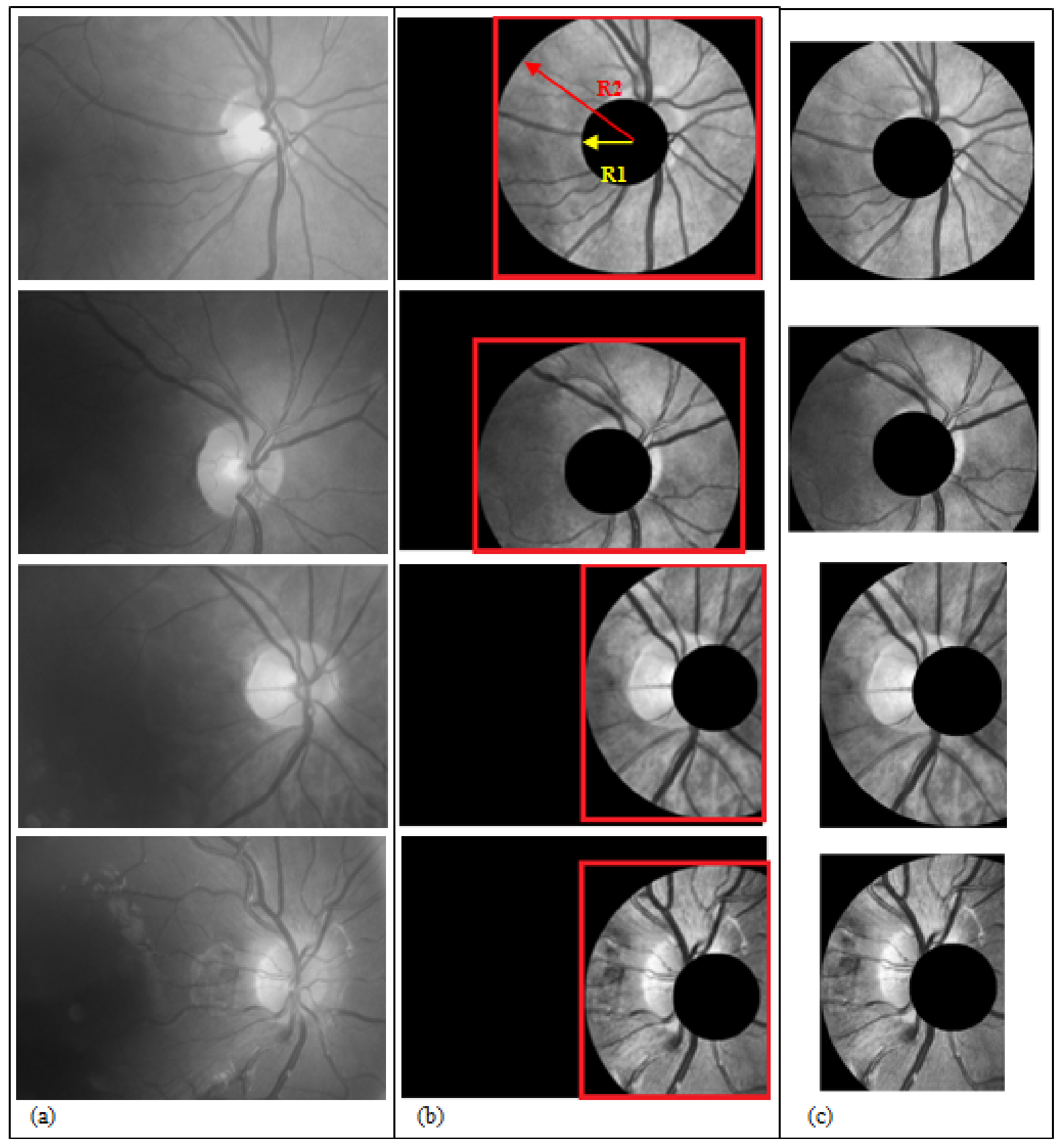

Fig. 3. Optical disc Interest Ring detection : (a) The input retinal images, (b) Inhanced images with the localized Interest Ring (R1,R2) and (c) Final results of the proposed ODR method.

In our proposal, the adaptive contrast histogram equalization technique [7] is applied to retinal grayscale images to perform the contrast enhancement in order to remove these imperfections. This technique enhances the contrast adaptively across the image by limiting the maximum slope in the transformation function. Instead of applying the histogram equalization on the entire image, it is applied only on small tiles in the image. After that, the neighboring tiles are combined using bilinear interpolation to reduce induced boundaries. Figure 2 illustrates the histograms of the input retinal image an the enhanced one.

\section{B. Optical disc Detection}

The optical disc localization follows two steps: First, the Sauvola binarization method [4] takes the grayscale enhanced image as input to separate brighter regions from dark background. In this algorithm, a threshold $\mathrm{T}$ at each pixel is computed with the dynamic range $(\mathrm{R})$ of the standard deviation using the equation (1):

$$
T=m(1+k s /(R-1))
$$


Where $\mathrm{m}$ and $\mathrm{s}$ are respectively the mean and standard deviation of a given window centered on the current pixel and $\mathrm{k}$ is a fixed value.

At the next step, we compute the Euclidean distance of the binary image. For each pixel in this image, the distance transform assigns a number that is the distance between that pixel and the nearest non zero one in the image. Finally, we compute the minimum of the resultant image and we localize its coordinates $(\mathrm{Cx}, \mathrm{Cy})$ which refer to the center of the most brilliant region, ie the optical disc.

\section{Interest Ring Extraction around the optical disc}

This step aimed to restrict our interest region around the detected center. Vessels around the optical disc are more important for identification purposes because they contain the high density of information. In fact, on one hand inside the optical disc, vessels are less contrasted and noisy. On the other hand, if they are farther from the optical disc, they become thinner and their distribution is more arbitrary. So, we propose to focus on vessels in the neighborhood of the optical disc. We extract a ring centered at the detected center, with radius $\mathrm{R} 1$ and $\mathrm{R} 2(R 1<R 2)$ as an interest region of retinal image. Finally, as shown in figure 3, we extract the rectangle around the biggest circle (R2) to be used as input for feature extraction step. Actually, the choice of R1 and R2 values affects significantly the rate of personal identification. In fact, the bigger R2 value, the higher personal recognition rate is. However, the needed computation time will be high too. So, in this work, an optimal combination of R1 and R2 is chosen through apprenticeship as follow:

$$
\begin{aligned}
& R 2=N / 2 \\
& R 1=N / 6
\end{aligned}
$$

Where $\mathrm{N}$ is the width of the input retinal image.

\section{EXPERIMENTAL RESULTS AND DISCUSSION}

In order to evaluate the efficiency of our proposed preprocessing method, we integrate it into a personal identification system. The assessed system is composed of three main phases: our proposed ODR preprocessing, the Scale Invariant Feature Transform (SIFT) [15] extraction which extract local descriptors invariant to geometric transformation such as rotation, translation and illumination variations, and the test g2NN of Lowe [15] feature matching in order to find the number of matched pairs between retinal images. All the experiments are implemented in MATLAB, and performed on a PC with a 3.2 GHz CPU and $4 \mathrm{G}$ Byte memory. The method was applied on the VARIA database [16] which is built for evaluation of retinal recognition systems. This database includes 233 retinal images of 139 different subjects with a resolution of $768 *$ 584. The images have been acquired over a time span of several years with a TopCop NW-100 model non-mydriatic retinal camera. These images are optic disc centered and have a high variability in contrast and illumination, allowing the system to be assessed in quite hard conditions and simulating a more realistic environment.

To evaluate the efficiency of our proposed ODR based identification system in terms of quality, we compare our proposed system previously described with the same identification system without preprocessing and using the Top-hat Transform method [13].

The Identification Rate curves for these retinal biometric systems using the VARIA database are illustrated in the figure 4. We can see clearly that our system is the most stable one and identifies the higher rates.

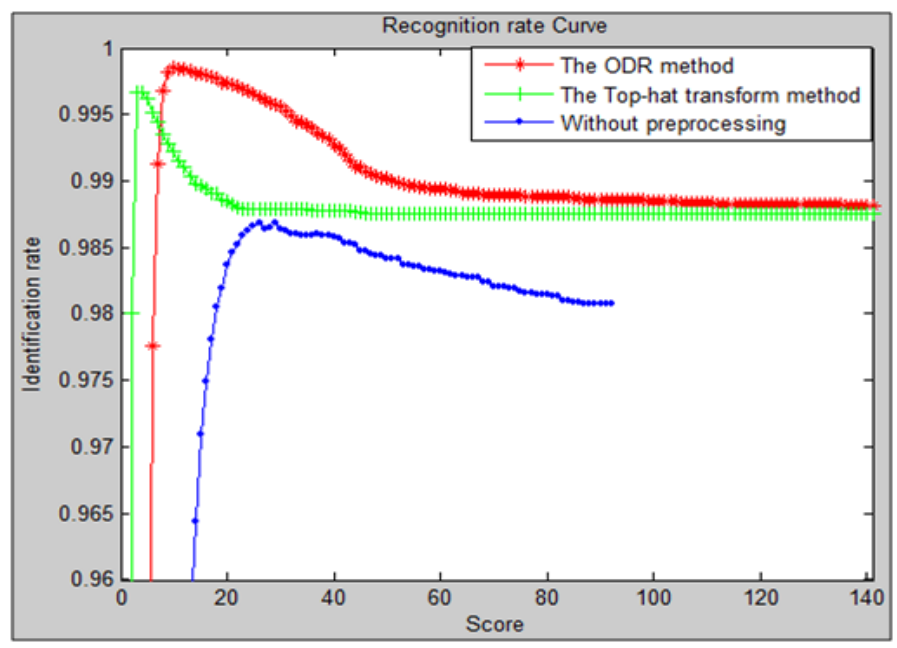

Fig. 4. The Identification Rate Curves of retinal Human identification system based on three preprocessing.

Table1 shows that our proposed system allows higher identification rate with $99.89 \%$ compared to the other methods.

Table 1. The average processing time and the Accuracy recognition rate of identification system based on different preprocessing methods.

\begin{tabular}{|l|cl|}
\hline Preprocessing method & $\begin{array}{c}\text { Processing time } \\
\text { (average) }\end{array}$ & $\begin{array}{l}\text { Accuracy } \\
\text { Identification } \\
\text { rate }\end{array}$ \\
\hline The ODR method & $10.3 \mathrm{~s}$ & $99.89 \%$ \\
Without preprocessing & $43.18 \mathrm{~s}$ & $98.6 \%$ \\
The Top-hat Transform method[13] & $232.13 \mathrm{~s}$ & $99.57 \%$ \\
\hline
\end{tabular}

The identification system without preprocessing step ensures only $98.6 \%$ due to the poor quality of input retinal images which generally suffer from low gray level contrast and illumination variations that can affects keypoints extraction and matching. Although applying the top-hat transform [13] as a preprocessing step, the identification rate has been improved from $98.6 \%$ to $99.57 \%$, it stills lower than our proposed system. Even if the proposed system improve slightly the identification rate compared to other systems, the most gain is in term of execution time. Indeed, as shown in table 1 , it has been seriously reduced from $232.13 \mathrm{~s}$ in retinal identification system with the Top-hat Transfom method and 42.18s without 
preprocessing to only 10 s with our ODR preprocessing. This reached execution time is essentially due the extraction of the interest ring around the optical disc. This helps to keep the most vessel information while reducing the extraction SIFT keypoints which decreases the processing time of our system.

In Identification mode of biometric system, there is an only error that can influence the obtained performance. This error is called The False Match Rate (FMR). It defines the probability that the system incorrectly matches the input pattern to a non-matching template in the database. In fact, It measures the percent of invalid input identities which are incorrectly assigned to the persons. As this error is little, the system is more efficient [17].

Table 2. The False Match Rate (FMR) of Identification system based on three preprocessing methods.

\begin{tabular}{|l|c|}
\hline Preprocessing method & False Match Rate \\
\hline The ODR method & $4.310^{-5}$ \\
The Top-hat Transform method [10] & $1.3210^{-4}$ \\
Without preprocessing & $1.610^{-3}$ \\
\hline
\end{tabular}

The table 2 illustrates the efficiency of our proposed ODR based system with the less FMR rate compared to other identification systems. Indeed, the FMR of our proposed system reach $4.310^{-5}$ which is very low compared to 1.32 $10^{-4}$ in the top-hat transfom based system and $1.610^{-3}$ in the identification system without prepocessing.

\section{CONCLUSION}

In this paper, we have presented a robust retinal identification approach based on Optical Disc Interest Ring detection. This method consists of three stages: Image Enhancement, optical disc detection and Interest Ring Extraction around the optical discs center. Conducted experiments prove that the proposed ODR method is suitable for the preprocessing of retinal images in order to reduce the false rate of human identification. In order to evaluate the efficiency of our method, we compare it with the Top-hat Transform. Obtained results show that our ODR preprocessing allows improving the identification performance to reach $99.89 \%$ in term of Identification rate, while reducing seriously the total execution time compared to other systems.

\section{REFERENCES}

[1] Biometrics Consortium homepage. http://www.biometrics.org

[2] Yong Z., Tieniu T., YunHong W. Biometric personal identification based on iris patterns 15th ICPR,Barcelone, Espagne, 2000.

[3] B.N Chandrasheckar, B. Honnaraju. Human recognition System based on Retina Vascular Network Characteristics.

[4] J. Sauvola, M. Pietikinen. Adaptive document image binarization, Pattern Recognition. Pattern Recognition, Volume 33, Issue 2, Pages 225-236 ISSN 0031-3203, February 2000.

[5] Christel -Loc TISSE Contribution la vrification biomtrique de personnes par reconnaissance de liris. $\mathrm{PhD}$ thesis, Montpellier II university, Montpellier II-France, Octobre 2003.

[6] Marn, D., Aquino, A., Gegndez-Arias, M.E.; Bravo, J.M. A new supervised method for blood vessel segmentation in retinal images by using gray-level and moment invariants-based features. IEEE Trans. Med. Imaging, 30, 146158, 2011.
[7] J.Tang, E.Peli and S.Acton. Image enhancement using a contrast measure in the compressed domain. IEEE Signal Processing Letters, 10(110):289292, October 2003.

[8] Hannu Oinonen, Heikki Forsvik, Pekka Ruusuvuori, Olli Yli-Harja, Ville Voipio, and Heikki Huttunen. Identity verification based on vessel matching from fundus images. In ICIP, page 4089-4092. IEEE, 2010.

[9] J. Hajer and H.Kamel Caractrisation de la rtine en vue de llaboration dune mthode biomtrique didentification de personnes. In SETIT, 3rd International Conference: Sciences of Electronic, Technologies of Information and Telecom.,March 27-31, 2005.

[10] H. Tabatabaee, A. Milani-Fard, and H. Jafariani. A Novel Human Identifier System Using Retina Image and Fuzzy Clustering Approach. In in Proceedings of the 2nd IEEE International Conference on Information and Communication Technologies (ICTTA 06),pp. 1031-1036, Damascus, Syria, April 2006.

[11] M. Ortega, C. Marino, M. G. Penedo, M. Blanco, F. Gonzalez. Biometric Authentification Using Digital Retinal Images. In in Proceedings of the 5th WSEAS International Conference on Applied Computer Science (ACOSO6),pp. 422427, Hangzhou, China, April 2006.

[12] A.F. Frangi, R.F. Frangi,W.J. Niessen, K.L Vincken, and M.A. Viergever. Multiscale vessel enhancement filtering. In in Image Computing and Computer-Assisted Interventation MICCAI98, pp. 130137. SpringerVerlag, 1998.

[13] Xianjing Meng, Yilong Yin, Gongping Yang and Xiaoming Xi. Retinal Identification Based on an Improved Circular Gabor Filter and Scale Invariant Feature Transform. In Sensors (14248220), Vol. 13 Issue 7, p9248, Jul 2013.

[14] H. Farzin, H.A. Moghaddam and M.S. Moin. A Novel Retinal Identification System. In EURASIPJournal on Advances in Signal Processing, vol. 2008, no. Article ID:280635, 2008.

[15] David G. Lowe Distinctive Image Features from Scale-Invariant Keypoints. Computer Science Department, University of British Columbia, Vancouver, B.C., Canada, January 5, 2004.

[16] VARIA. VARPA. Retinal Images for Authentification (Database) http://www.varpa.es/varia.html.

[17] Wayman, J.L. Error Rate Equations for the General Biometric System. In IEEE Robotics Automation, 35-48, 6, 9 Jan. 1999. 